\title{
Reversible conformation-driven order-order transition of peptide-mimic poly( $n$-alkyl isocyanate) in thin films via selective solvent-annealing
}

\author{
Yecheol Rho',4, Joonkeun Min ${ }^{2,4}$, Jinhwan Yoon ${ }^{3,4}$, Byungcheol Ahn ${ }^{1}$, Sungmin Jung1, Kyungtae Kim \\ Priyank N Shah ${ }^{2}$, Jae-Suk Lee ${ }^{2}$ and Moonhor Ree ${ }^{1}$
}

We report for the first time the conformational and structural details of peptide-mimic poly(n-hexyl isocyanate) (PHIC). PHIC is a representative poly(n-alkyl isocyanate)s, which have received significant attention because of their unique stiff chain characteristics and potential applications in various fields. A well-ordered hexagonal close packing structure of PHIC with $\mathbf{8}_{3}$ helical conformation was clearly observed in the nanoscale thin films that were selectively annealed with carbon disulfide $\left(\mathrm{CS}_{2}\right)$. A well-ordered multi-bilayer structure of the polymer with $\beta$-sheet conformation was also clearly formed in the films that were selectively annealed with toluene. In addition, a fully reversible transformation between these two self-assembled structures was demonstrated by consecutive annealings with $\mathrm{CS}_{2}$ and toluene.

NPG Asia Materials (2012) 4, e29; doi:10.1038/am.2012.54; published online 26 October 2012

Keywords: conformation change; hexagonal close packing structure; multi-bilayer structure; peptide-mimic poly $(n$-alkyl isocyanate); reversible order-order transition; selective solvent annealing; self-assembly

\section{INTRODUCTION}

Poly( $n$-alkyl isocyanate)s (PAICs) have received considerable attention as helical, stiff, rod-like polymers because of their unique characteristics and structural features, which include their large persistence length $(20-60 \mathrm{~nm})$ and their potential applications in chiral recognition, optical switches, liquid crystals, degradable materials and composites. ${ }^{1-8}$ Considerable research efforts have been dedicated to improving the understanding of the conformation and packing of PAICs. Specifically, $\operatorname{poly}(n$-butyl isocyanate) (PBIC) and poly ( $n$-hexyl isocyanate) (PHIC) (Figure 1a) have been thoroughly investigated as representative PAICs. Despite these extensive studies, however, the conformation and molecular packing of PAICs have yet to be unequivocally established. For example, several helical conformations have been proposed, namely $2{ }_{1}, 5_{1}$ and $8_{3}$ in solution ${ }^{9-12}$ and $8_{3}, 8_{5}$ and $12_{5}$ in the solid state. ${ }^{13-15}$ Furthermore, triclinic, monoclinic and pseudo-hexagonal lattices have all been proposed as possible molecular packing structures. ${ }^{13,14,16}$

In the present work, we provide the first report on the formation of PHIC in thin films with both a helical conformation-based hexagonal packing order and a $\beta$-sheet conformation-based lamellar structure; we also demonstrate the complete reversibility of the order-order phase transition under selective solvent annealing.

\section{MATERIALS AND METHODS}

Materials and PHIC synthesis

PHIC was synthesized from $n$-hexyl isocyanate using living anionic polymerization based on a method reported in the literature. ${ }^{17,18} n$-Hexyl isocyanate (HIC, 97\%, Aldrich, Milwaukee, WI, USA) was dried over $\mathrm{CaH}_{2}$ and distilled under vacuum. An initiator, sodium benzanilide (Na-BA) in tetrahydrofuran (THF, $50 \mathrm{ml}$ ), was prepared from the reaction of equivalent amounts of benzanilide $(8.70 \mathrm{~g}, 0.043 \mathrm{~mol})$ and elemental sodium $(1.00 \mathrm{~g}, 0.043 \mathrm{~mol})$ at room temperature. The polymerization reactions of HIC were conducted under high vacuum in a glass apparatus equipped with break-seals. In a typical procedure, the initiator solution, Na-BA $(0.013 \mathrm{~g}, 0.066 \mathrm{mmol})$ in THF, was transferred into the reaction flask through the break-seal, and the solution temperature was then equilibrated to the reaction temperature of $-98^{\circ} \mathrm{C}$. The polymerization was initiated by adding the HIC monomer $(0.753 \mathrm{~g}, 5.93 \mathrm{mmol})$ in THF to the initiator solution. The reaction was terminated after $60 \mathrm{~min}$ by adding a 20-fold excess of $\mathrm{HCl}$ in methanol when the target PHIC product was precipitated. The PHIC precipitates were filtered off and dried in vacuo.

${ }^{1}$ Department of Chemistry, Division of Advanced Materials Science, Pohang Accelerator Laboratory, Centre for Electro-Photo Behaviours in Advanced Molecular Systems, BK School of Molecular Science and Polymer Research Institute, Pohang University of Science and Technology, Pohang, Republic of Korea; ${ }^{2}$ Department of Nano Bio Materials and Electronics, School of Materials Science and Engineering, Gwangju Institute of Science and Technology, Gwangju, Republic of Korea and ${ }^{3}$ Department of Chemistry, Dong-A University, Busan, Republic of Korea

${ }^{4}$ These authors contributed equally to this paper.

Correspondence: Professor M Ree, Department of Chemistry, Division of Advanced Materials Science, Pohang Accelerator Laboratory, and Centre for Electro-Photo Behaviours in Advanced Molecular Systems, Pohang University of Science and Technology, Pohang 790-784, Republic of Korea.

E-mail: ree@postech.edu

or Professor J-S Lee, Department of Nano Bio Materials and Electronics, School of Materials Science and Engineering, Gwangju Institute of Science and Technology, Gwangju 500-712, Republic of Korea.

E-mail: jslee@gist.ac.kr

Received 16 July 2012; revised 4 September 2012; accepted 6 September 2012 


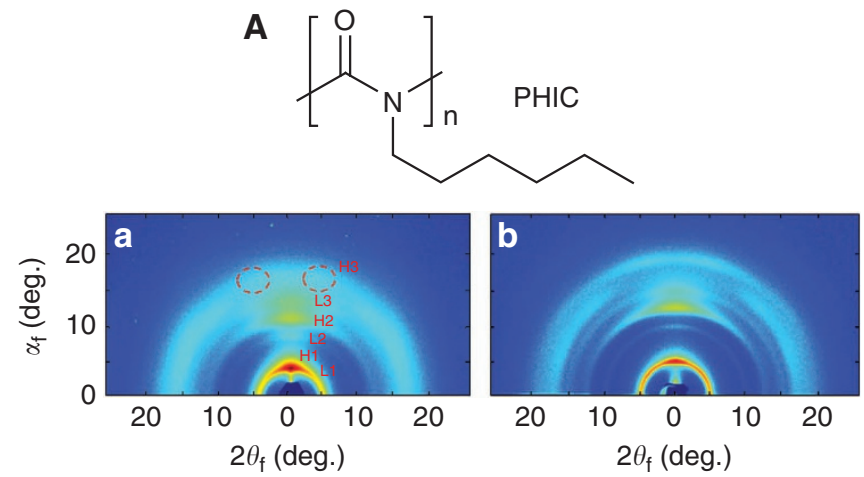

Figure 1 Two-dimensional (2D) GIXS patterns measured at $\alpha_{i}=0.150$ for thin films of PHIC (A) deposited onto silicon substrates: (a) as-cast; (b) $\mathrm{CHCl}_{3}$-annealed for $3 \mathrm{~h}$.

The methanol soluble portion was determined quantitatively by weighing the residue after evaporation of methanol and using proton nuclear magnetic resonance spectroscopy to determine whether any unreacted monomers and/ or trimers were present. The yield of the polymer was 99\%. The obtained PHIC product was characterized using both proton and carbon nuclear magnetic resonance $\left({ }^{1} \mathrm{H}\right.$ and ${ }^{13} \mathrm{C}$ NMR) spectroscopies with a JEOL NMR spectrometer (model JNM-LA300WB, JEOL Ltd, Tokyo, Japan) and using Fourier transform infrared (FT-IR) spectroscopy with a Perkin Elmer spectrometer (model 2000, Perkin Elmer, Waltham, MA, USA). PHIC ${ }^{1} \mathrm{H}$ NMR $\left(\mathrm{CDCl}_{3}, 300 \mathrm{MHz}\right), \delta$ (p.p.m.): $0.9\left(3 \mathrm{H}, \mathrm{CH}_{3}\right), 1.0-2.0\left(8 \mathrm{H},\left(\mathrm{CH}_{2}\right)_{4}\right), 3.7(2 \mathrm{H}$, $\mathrm{N}-\mathrm{CH}_{2^{-}}$) (Supplementary Figure $\mathrm{S} 1$ ). ${ }^{13} \mathrm{C} \mathrm{NMR}\left(\mathrm{CDCl}_{3}, 75 \mathrm{MHz}\right), \delta$ (p.p.m.): $\left.14.5\left(\mathrm{CH}_{3}\right), 22.5\left(\mathrm{CH}_{2}\right), 26.2\left(\mathrm{CH}_{2}\right), 28.5\left(\mathrm{CH}_{2}\right), 31.5\left(\mathrm{CH}_{2}\right), 48.6\left(\mathrm{~N}_{-} \mathrm{CH}_{2}\right)^{-}\right)$, $156.8(C=\mathrm{O})$ (Supplementary Figure S2). IR $\left(\mathrm{KBr}, \mathrm{cm}^{-1}\right): 3441(-\mathrm{NH}), 2959$, 2932, $2860\left(\mathrm{CH}_{2}\right), 1700(\mathrm{C}=\mathrm{O}), 1349 / 1297$ (disubstituted amide), 1227, $1175,1092,785,728\left(\mathrm{CH}_{2}\right)$.

The molecular weights of the polymers were determined using a multi-angle laser light scattering detector system and a size exclusion chromatography system (Interferometric Refractometry 478-009-690, Optilab DSP, Phoeix, AZ, USA and DAWN EOS Laser Photometer 113-E, Wyatt Technology, Santa Barbara, CA, USA) with four columns (HR 0.5, HR 1, HR 3 and HR 4, Waters Styragel columns run in series with column pore sizes of 50, 100, 500 and $1000 \AA$, respectively). THF with triethylamine (to prevent the adsorption of the hydrophilic polymer on the column) was used as the mobile phase at a flow rate of $1.0 \mathrm{ml}$ per min. The $d n / d c$ values for the polymers in THF at $40{ }^{\circ} \mathrm{C}$ were measured with an LED (Optilab DSP) source. After the $d n / d c$ values were measured for five different concentrations for the PHIC sample, size exclusion chromatography -multi-angle laser light scattering detector system data were obtained using refractive index detection at $40^{\circ} \mathrm{C}$.

\section{Thin film preparation}

PHIC solutions ( $1 \mathrm{wt} \%$ polymer) were prepared in chloroform $\left(\mathrm{CHCl}_{3}\right)$ and then spin-coated onto silicon substrates at 2000 r.p.m. for 40 s, which was followed by drying under vacuum at room temperature for $12 \mathrm{~h}$. The obtained thin films (60-70 nm thick) were then annealed at room temperature under $\mathrm{CHCl}_{3}$, carbon disulfide $\left(\mathrm{CS}_{2}\right)$, or toluene vapor. The $\mathrm{CHCl}_{3}$-annealing was conducted for $3 \mathrm{~h}$. The $\mathrm{CS}_{2}$ annealing was performed for $1 \mathrm{~h}$, whereas the toluene annealing was conducted for $5 \mathrm{~h}$. After this step, the solvent-annealed films were dried under vacuum at room temperature for 1 day. These solventannealing and subsequent drying steps were repeated one or two times for some of the first solvent-annealed films by changing the solvent $\left(\mathrm{CS}_{2}\right.$ or toluene); for example, some of the 1 st $\mathrm{CS}_{2}$-annealed films were subsequently annealed with toluene, whereas some of the first toluene-annealed films were then annealed with $\mathrm{CS}_{2}$.

\section{Synchrotron grazing incidence X-ray scattering (GIXS)} measurements

GIXS measurements were performed at beamline $3 \mathrm{C}^{19-23}$ of the Pohang Accelerator Laboratory at the Pohang University of Science and Technology.
Table 1 Scattering peaks and their $d$-spacings from the 2D-GIXS patterns measured for the as-cast and $\mathrm{CHCl}_{3}$-annealed $\mathrm{PHIC}$ films

\begin{tabular}{lcccccc}
\hline Scattering peaks & $L 1$ & $H 1$ & $L 2$ & $H 2$ & $L 3$ & $H 3$ \\
\hline$\alpha_{\mathrm{f}}, 2 \theta_{\mathrm{f}}$ (deg.) & $4.84,0$ & $5.93,0$ & $9.67,0$ & $11.89,0$ & $14.52,0$ & $17.38,4.46$ \\
$d$-spacing (nm) & 1.63 & 1.33 & 0.82 & 0.67 & 0.55 & 0.45 \\
\hline
\end{tabular}

Abbreviations: GIXS, grazing incidence X-ray scattering; PHIC, poly(n-hexyl isocyanate).

The diffraction patterns were recorded at a sample-to-detector distance of $123 \mathrm{~mm}$ with an X-ray wavelength of $\lambda=0.138 \mathrm{~nm}$ using a two-dimensional (2D) charge-coupled detector (Mar, USA). The samples were mounted on a homemade $\mathrm{z}$ axis goniometer equipped with a vacuum. The incidence angle, $\alpha_{i}$, of the X-ray beam was set at $0.150^{\circ}$, which is between the critical angles of the films and the silicon substrate; with this $\alpha_{i}$ value, the X-ray beam can penetrate into the entire polymer film layer. All of the GIXS measurements were conducted at $25^{\circ} \mathrm{C}$. Each diffraction pattern was collected for $30 \mathrm{~s}$. The measured scattering data were quantitatively analyzed using the GIXS formulas derived for several structural models, including hexagonally close packed cylinders and a multilayer structure (Supplementary Information).

\section{RESULTS AND DISCUSSION}

NMR and IR spectroscopic analyses confirmed that the PHIC polymer was successfully synthesized. The number average molecular weight and polydispersity index of the polymer were determined to be 61000 and 1.20 , respectively.

The structures of the solvent-annealed polymer films were examined using GIXS with a synchrotron radiation source. The scattering pattern of the as-cast PHIC film was complicated and weak in intensity (Figure 1a). A similar scattering pattern was observed for the film that underwent subsequent annealing under $\mathrm{CHCl}_{3}$ vapor (Figure 1b); however, the scattering peaks were enhanced in intensity and sharpened in shape. The scattering pattern exhibited periodic anisotropic rings (marked with L1, L2 and L3 in Figure 1a; Table 1) along the $\alpha_{\mathrm{f}}$ direction. The positions of these rings relative to the specular reflection position were 1,2 and 3, and their scattering angles were $4.84^{\circ}, 9.67^{\circ}$ and $14.52^{\circ}$, respectively. This scattering feature indicated the presence of lamellae stacked along a direction normal to the film plane. The film exhibited three additional arc-like spots at $\alpha_{\mathrm{f}}=5.93^{\circ}, 11.89^{\circ}$ and $17.38^{\circ}$ (marked with $\mathrm{H} 1, \mathrm{H} 2$ and $\mathrm{H} 3$, respectively, in Figure 1a; Table 1), which suggests the presence of a poorly developed ordered structure in the film in addition to the lamellar structure described above. However, drawing any firm conclusions about an exact ordered structure from such weak, broad scattering peaks is difficult. Overall, both the as-cast film and the $\mathrm{CHCl}_{3}$-annealed film presented at least two different molecular packing orders with different chain conformations. The degree of ordering and the fraction of the film composed of these structures (namely, a lamellar structure and another ordered structure) were relatively low in both films; the degree of ordering was significantly lower in the as-cast film than in the $\mathrm{CHCl}_{3}$-annealed film, as was the fraction of the film occupied by the structures in question.

Surprisingly, the $\mathrm{CS}_{2}$-annealed film demonstrated a very clean, crystal-like scattering pattern (Figure $2 \mathrm{a}$ ). The pattern clearly exhibited several scattering spots, with regular spacings of $<15^{\circ}$. These scattering spots satisfied the Bragg conditions for a hexagonal reciprocal lattice of cylinders lying in the film plane. Some representative scattering spots were assigned (Supplementary Figure S3). The scattering spots at $\alpha_{\mathrm{f}}=5.93^{\circ}$ and $11.87^{\circ}$ along the $\alpha_{\mathrm{f}}$ direction, at $2 \theta_{\mathrm{f}}=0^{\circ}$, were indexed as the reflection peaks of the $(01)$ and $(02)$ planes, respectively. The $d$-spacing was determined to be $1.34 \mathrm{~nm}$ for the first-order spot and $0.67 \mathrm{~nm}$ for the second order spot. 

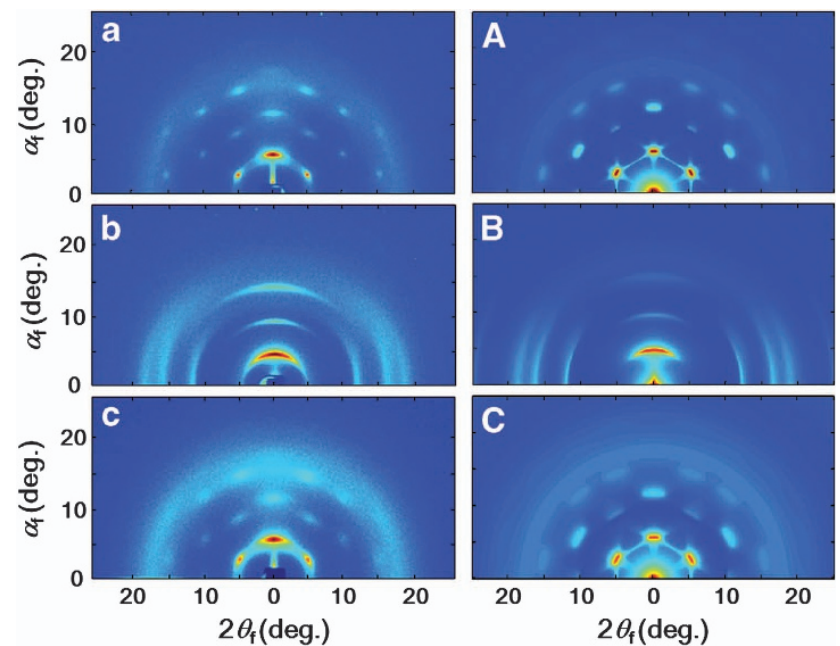

Figure 2 2D-GIXS patterns measured at $\alpha_{\mathrm{i}}=0.150^{\circ}$ for $\mathrm{PHIC}$ thin films deposited onto silicon substrates: (a) $\mathrm{CS}_{2}$-annealed for $1 \mathrm{~h}$; (b) tolueneannealed for $5 \mathrm{~h}$ after $\mathrm{CS}_{2}$-annealing; (c) $\mathrm{CS}_{2}$-annealed for $1 \mathrm{~h}$ after $\mathrm{CS}_{2}$-and toluene annealing in sequence. Scattering patterns reconstructed from the determined structural parameters (Tables 2 and 3 ) using the GIXS formula: (A) HCP structure determined from an analysis of the pattern in (a); (B) multibilayer structure determined from an analysis of the pattern in panel (b); (C) HCP structure determined from an analysis of the pattern in panel (c).

Two additional scattering spots (one at $\alpha_{\mathrm{f}}=2.92^{\circ}$ and $2 \theta_{\mathrm{f}}=5.04^{\circ}$ and the other at $\alpha_{\mathrm{f}}=5.85^{\circ}$ and $2 \theta_{\mathrm{f}}=9.97^{\circ}$ ) were assigned as the reflection peaks of the (10) and (20) planes, respectively. The $d$-spacings of these reflections were determined to be 1.34 and $0.67 \mathrm{~nm}$, respectively. Here, the $d$-spacing of $1.34 \mathrm{~nm}$ corresponded to the mean interdistance between the cylinder layers in the hexagonal structure. The scattering spot at $\alpha_{\mathrm{f}}=8.90^{\circ}$ and $2 \theta_{\mathrm{f}}=5.04^{\circ}$ was indexed as the reflection peak of the (11) plane, and its $d$-spacing was determined to be $0.77 \mathrm{~nm}$. From these results, the mean interdistance of the cylinders was estimated to be $1.55 \mathrm{~nm}$. In addition, the scattering pattern contained a weak, broad, isotropic scattering ring at $17.70^{\circ}$. The $d$-spacing of this ring was estimated to be $0.45 \mathrm{~nm}$. Considering an in-plane oriented hexagonal packing order of PHIC polymer chains, this isotropic scattering ring can only originate from the $n$-hexyl side groups along the individual polymer chain backbones in a helical conformation. The $n$-hexyl groups in the nearest neighboring positions might have the highest population, which exhibits a maximum intensity in the isotropic scattering ring. Taking this situation into consideration, the $d$-spacing $(=0.45 \mathrm{~nm})$, which was determined from the peak maximum of the isotropic scattering ring, can be assigned to the mean interdistance of the nearest neighbored $n$-hexyl groups along the single-PHIC polymer chain in a helical conformation (which is a structural member of the in-plane oriented hexagonal packing order). Considering this fact, we performed calculations on all of the possible helical conformations $\left(2_{1}, 5_{1}, 8_{5}, 12_{5}\right.$ and so on) for the PHIC polymer chain using the Cerius ${ }^{2}$ software package (Accelrys, San Diego, CA, USA). The resulting $d$-spacing value $(=0.45 \mathrm{~nm})$ of the isotropic scattering ring at the maximum intensity is very similar to the interdistance of the neighboring $n$-hexyl side groups in the PHIC chain with the $8_{3}$ helical conformations, rather than any other conformations. This result is, for the first time, direct experimental evidence for the helical conformation of PHIC chains in a hexagonal packing structure. Furthermore, the results also confirmed that synchrotron GIXS is a very unique, powerful technique for determining polymer chain conformations and their hierarchical structures.

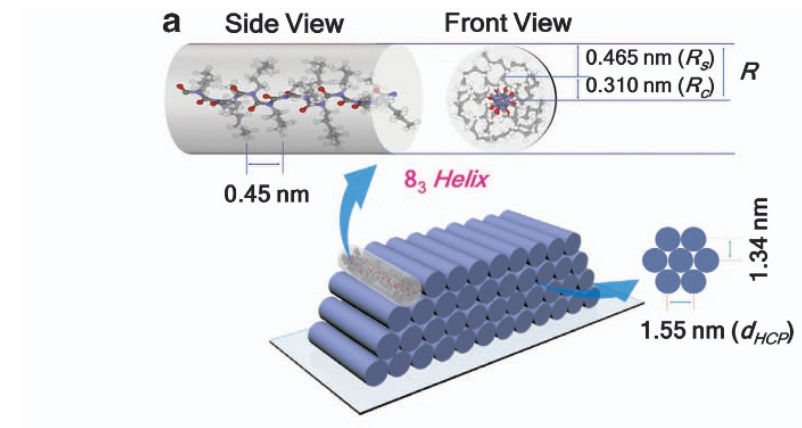

b
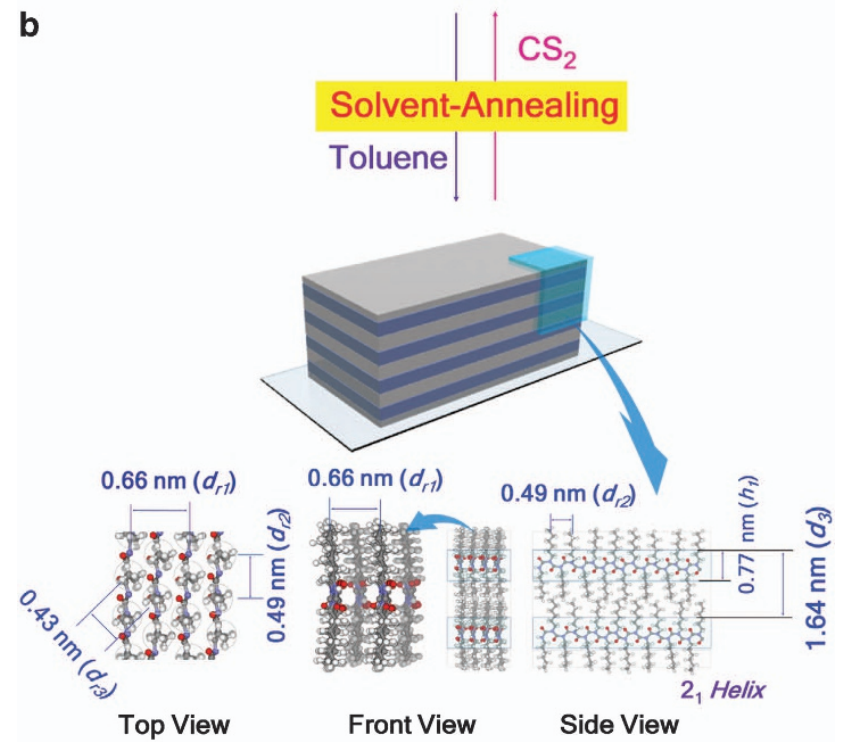

Figure 3 Molecular conformation and packing order of PHIC in thin films and their reversible transformations via selective solvent-annealing: (a) HCP structure with $8_{3}$ helical conformation; (b) molecular multi-bilayer structure with $\beta$-sheet conformation.

With the above information, the 2D-scattering pattern (Figure 2a) was quantitatively analyzed using the GIXS formula for a hexagonal close packing (HCP) structure model (Figure 3a and Supplementary Figure S4), which allowed all of the structural and orientation parameters to be determined. The results from the data analysis are summarized in Table 2 . The determined molecular chain conformation and HCP structure are shown in Figure 3a. This analysis confirmed that the $\mathrm{CS}_{2}$-annealed film formed a HCP structure where the molecular PHIC cylinders lie in the film plane. The lattice parameter $\left(d_{\mathrm{HCP}}\right)$ of the HCP structure was determined to be $1.55 \mathrm{~nm}$, which is the mean interdistance of the molecular PHIC cylinders. The length of the fully extended $n$-hexyl group was calculated to be $0.83 \mathrm{~nm}$ using the Cerius ${ }^{2}$ software package. Considering this side group length and the 83 conformations, the molecular PHIC cylinder was determined to have a diameter of $1.55 \mathrm{~nm}$. This cylinder diameter is the same as the lattice parameter, $d_{\mathrm{HCB}}$ of the HCP structure, which confirms that the molecular PHIC cylinders were closely packed with each other in the structure. Interestingly, the analysis revealed that the molecular PHIC cylinder consists of a relatively dense core region (whose radius, $R_{\mathcal{C}}$, is $0.310 \mathrm{~nm}$ ) and a less dense shell region with a thickness of $0.465 \mathrm{~nm}\left(R_{\mathrm{s}}\right)$; here, the core region was composed of the polymer backbone and the inner parts of the $n$-hexyl side groups, whereas the shell region consisted of the remaining parts of the side groups. 
Table 2 Structural parameters of the PHIC films (which were annealed with $\mathrm{CS}_{2}$ or consecutively annealed with $\mathrm{CS}_{2}$, toluene and $\mathrm{CS}_{2}$ ) determined by GIXS measurements and data analysis

Structural parameters

\begin{tabular}{|c|c|c|c|c|c|c|c|c|c|c|c|}
\hline PHIC films & $\mathrm{d}_{H C P}(n m)^{\mathrm{a}}$ & $\mathrm{R}(n m)^{\mathrm{b}}$ & $\mathrm{R}_{c}(n m)^{\mathrm{c}}$ & $\sigma_{R c}(n m)^{\mathrm{d}}$ & $\mathrm{R}_{s}(n m)^{\mathrm{e}}$ & $\sigma_{R s}(n m)^{f}$ & $\mathrm{~g}_{10}^{\mathrm{g}}$ & $\mathrm{d}_{r}(n m)^{\mathrm{h}}$ & $\varphi(\text { deg. })^{i}$ & $\sigma_{\varphi}(\text { deg. })^{j}$ & $\mathrm{O}_{s}^{\mathrm{k}}$ \\
\hline Films annealed with $\mathrm{CS}_{2}$ & 1.55 & 0.775 & 0.310 & 0.069 & 0.465 & 0.052 & 0.057 & 0.45 & 0 & 1.7 & 0.999 \\
\hline $\begin{array}{l}\text { Films annealed consecutively with } \mathrm{CS}_{2} \text {, } \\
\text { toluene and } \mathrm{CS}_{2}\end{array}$ & 1.55 & 0.775 & 0.310 & 0.069 & 0.465 & 0.052 & 0.070 & 0.45 & 0 & 2.5 & 0.997 \\
\hline
\end{tabular}

Abbreviations: $\mathrm{CS}_{2}$, carbon disulfide; GIXS, grazing incidence X-ray scattering; PHIC, poly(n-hexyl isocyanate).

a Mean lattice dimension of the HCP structure, which is same with the mean interdistance of molecular PHIC cylinders (that is, helical polymer chains).

${ }^{b}$ Mean radius of the core-shell PHIC cylinders in the HCP structure: $R=R_{\mathrm{c}}+R_{\mathrm{s}}$.

${ }^{c}$ Mean radius of the core part of the core-shell PHIC cylinder.

${ }^{d}$ s.d. for the mean radius of the core part of the core-shell PHIC cylinder.

e Mean thickness of the shell part of the core-shell PHIC cylinder.

fs.d. for the mean thickness of the shell part of the core-shell PHIC cylinder.

gPositional distortion factor of the molecular PHIC cylinder (that is, paracrystal). In this work, isotropic distortion was assumed (that is, $g_{10}=g_{11}=g_{01}$ ).

${ }^{h}$ Mean interdistance between the nearest $n$-hexyl side groups along the helical PHIC polymer chain.

iPolar angle (that is, orientation angle) between the orientation $\mathbf{n}$ vector of the hexagonal close packed structure and the out-of-plane direction of the film.

s.d. for the orientation angle.

Orientational order parameter.

Table 3 Structural parameters of the PHIC films consecutively annealed with $\mathrm{CS}_{2}$ and toluene, which were determined by GIXS measurements and data analysis

Structural parameters

\begin{tabular}{|c|c|c|c|c|c|c|c|c|c|c|}
\hline PHIC film & $\mathrm{d}_{3}(n m)^{\mathrm{a}}$ & $\mathrm{h}_{1}(n m)^{\mathrm{b}}$ & $\sigma_{h 1}(n m)^{\mathrm{c}}$ & $g_{33^{d}}$ & $g_{r r}{ }^{e}$ & $\mathrm{~d}_{r 1}(n m)^{f}$ & $\mathrm{~d}_{r 2}(n m)^{\mathrm{g}}$ & $\varphi(\text { deg. })^{h}$ & $\sigma_{\varphi}(\text { deg. })^{i}$ & $\mathrm{O}_{s}{ }^{j}$ \\
\hline Films annealed consecutively with $\mathrm{CS}_{2}$ and toluene & 1.64 & 0.77 & 0.13 & 0.052 & 0.048 & 0.66 & 0.49 & 0 & 9.1 & 0.963 \\
\hline
\end{tabular}

Abbreviations: $\mathrm{CS}_{2}$, carbon disulfide; GIXS, grazing incidence X-ray scattering; PHIC, poly(n-hexyl isocyanate).

aLong period of multi-bilayer structure (that is, lamellar structure).

bMean thickness of a relatively dense sublayer in the lamella.

${ }^{c}$ s.d. for the mean thickness $h_{1}$ of the relatively dense sublayer in the lamella.

dPositional distortion factor of the fundamental vector $\mathbf{a}_{3}$ in the direction of $a_{3}$

epositional distortion factor of the fundamental vectors $a_{1}$ and $a_{2}$ in the direction $r$; here $r$ denotes the direction transverse (normal) to $a_{3}$.

${ }^{f}$ Mean interdistance between the $n$-hexyl side groups of the neighboring polymer chains whose repeat units are matched in position along their backbones.

gMean interdistance between the nearest $n$-hexyl side groups along the polymer backbone.

${ }^{\mathrm{h}}$ Polar angle (that is, orientation angle) between the orientation $\mathbf{n}$ vector of the multi-bilayer structure and the out-of-plane direction of the film.

's.d. for the orientation angle $\varphi$.

orientational order parameter.

Furthermore, the results collectively revealed that no interdigitation occurred between the neighboring, contacting molecular cylinders. In addition, the helical molecular cylinders were observed to have very small values for the positional distortion factors $\left(g_{10}=g_{11}=\right.$ $\left.g_{01}=0.057\right)$, which indicates that all of the helical molecular PHIC cylinders are stably positioned in the HCP structure. For the HCP structure, the orientational order parameter, $O_{s}$, was determined (with respect to the normal to the film plane) to be 0.999 , thereby confirming that the helical molecular PHIC cylinders of the HCP structure are almost completely lying in the film plane. From the structural model with these parameters, a scattering pattern was reconstructed using the GIXS formula; this pattern matched well with the measured scattering pattern (Figures $2 \mathrm{a}$ and $\mathrm{A}$ ).

The $\mathrm{CS}_{2}$-annealed films were subsequently annealed under toluene vapor. The toluene-annealed films were examined using GIXS. Surprisingly, these films also exhibited a 2D-scattering pattern, but it was considerably different from the pattern observed for the $\mathrm{CS}_{2}$-annealed films. The scattering pattern was observed to be identical to that of the as-cast films that were annealed with toluene. The films exhibited periodic arc peaks with regular spacing along the $\alpha_{\mathrm{f}}$ direction at $2 \theta_{\mathrm{f}}=0^{\circ} ; \alpha_{\mathrm{f}}=4.84^{\circ}, 9.67^{\circ}$, and $14.52^{\circ}$ (Figure $2 \mathrm{~b}$ ). The appearance of these scattering peaks suggests that a lamellar structure is present in the film and that its lamellae are stacked along a direction normal to the film plane. The $d$-spacing of the first-order arc peak was determined to be $1.64 \mathrm{~nm}$, which was larger than the diameter of the $8_{3}$ helical conformational polymer chain but was very close to twice the length $(0.83 \mathrm{~nm})$ of the fully extended $n$-hexyl group. Considering a lamellar structure, the $d$-spacing value may correspond to the long period (that is, the layer thickness). The scattering pattern exhibited additional arc peaks along the $2 \theta_{\mathrm{f}}$ direction at $\alpha_{\mathrm{f}}=0^{\circ} ; 2 \theta_{\mathrm{f}}=12.07^{\circ}, 16.32^{\circ}$ and $18.53^{\circ}$, which corresponded to $d$-spacings of $0.66,0.49$ and $0.43 \mathrm{~nm}$, respectively. These peaks may have resulted from the lateral packing of the polymer backbones and the side groups.

Considering these results, the scattering pattern was analyzed in detail using the GIXS formula for a molecular multi-bilayer structure model (Figure $3 \mathrm{~b}$ and Supplementary Figure S4). The results of the analysis are summarized in Table 3 . The determined structural model is shown in Figure $3 \mathrm{~b}$. The 2D-scattering pattern reconstructed with the structural model was confirmed to match well with the measured pattern (Figures $2 \mathrm{~b}$ and B). Collectively, these results indicate that the toluene-annealed film consisted of a well-ordered molecular multibilayer structure of the fully extended polymer chains in the $22_{1}$ conformation with no interdigitation between the side groups of adjacent molecular layers. For the multi-bilayer structure, the orientational order parameter, $O_{s}$, was determined (with respect to the normal to the film plane) to be 0.963 , which indicates that the layers were preferentially stacked in the out-of-plane direction of the film. In this structure, the long period, $d_{3}$, of the lamellae was determined to be a thickness of $1.67 \mathrm{~nm}$. Furthermore, the individual lamellae were composed of two sublayers, namely a relatively dense sublayer and a less dense sublayer; the dense sublayer (with a 
thickness of $0.77 \mathrm{~nm}\left(=h_{1}\right)$ ) consisted of the backbone and the inner parts of the side groups, whereas the less dense sublayer (with a thickness of $0.87 \mathrm{~nm}\left(=h_{2}\right)$ ) consisted of the remaining parts of the side groups from the adjacent two polymer chains (Figure $3 \mathrm{~b}$ ). This structural analysis also revealed a mean interdistance of $0.66 \mathrm{~nm}$ $\left(=d_{\mathrm{r} 1}\right)$ for the $n$-hexyl side groups between the neighboring polymer chains, whose repeat units were matched in position along their backbones (see the front and top views in Figure $3 \mathrm{~b}$ ); the mean interdistance of these side groups corresponded to the arc-scattering peak at $2 \theta_{\mathrm{f}}=12.07^{\circ}$ at $\alpha_{\mathrm{f}}=0^{\circ}$ (Figure 2b). In the multi-bilayer structure, the $n$-hexyl side groups along the backbone of the PHIC polymer chain were determined to have a lateral mean interdistance of $0.49 \mathrm{~nm}\left(=d_{\mathrm{r} 2}\right)$ (see the side and top views in Figure $3 \mathrm{~b}$ ), which corresponded to the arc-scattering peak that appeared at $2 \theta_{\mathrm{f}}=16.32^{\circ}$ at $\alpha_{\mathrm{f}}=0^{\circ}$ in Figure $2 \mathrm{~b}$. The side groups between the nearest neighboring polymer chains were observed to have a mean interdistance of $0.43 \mathrm{~nm}$ $\left(=d_{\mathrm{r} 3}\right)$ (see the top view in Figure $3 \mathrm{~b}$ ), which corresponded to the arcscattering peak at $2 \theta_{\mathrm{f}}=18.53^{\circ}$ at $\alpha_{\mathrm{f}}=0^{\circ}$ (Figure 2b); here, the repeat units of the nearest neighboring polymer chains were mismatched in position along their backbones. In addition, the $n$-hexyl side groups were determined to have very small values of positional distortion factors $\left(g_{33}=0.052\right.$ and $\left.g_{\text {rr }}=0.048\right)$, which reveals that the side groups were stably positioned in the out-of-plane and in-plane directions of the multi-bilayer structure. These results collectively demonstrate that the toluene-annealing process produced a complete phase transformation from a well-ordered HCP structure with $8_{3}$ helical conformational chains to a well-ordered multi-bilayer structure with $2_{1}$ helical (that is, $\beta$-sheet) conformational chains.

When the films were treated again with $\mathrm{CS}_{2}$ vapor, they presented a 2D-GIXS pattern (Figure 2c) similar to that (Figure 2a) observed for the $\mathrm{CS}_{2}$-annealed films before toluene annealing. The scattering peaks were slightly weakened in intensity; furthermore, the isotropic scattering ring at $17.70^{\circ}$ became slightly broader and stronger. The scattering pattern was analyzed in detail using a GIXS formula for the HCP structure (Supplementary Figure S4). The obtained structural parameters are listed in Table 2, which are almost identical to those determined for the films annealed with only $\mathrm{CS}_{2}$. From the obtained structural parameters, a scattering pattern was reconstructed using the GIXS formula. The calculated pattern matched well with the measured scattering pattern (Figures $2 \mathrm{c}$ and $\mathrm{C}$ ). These analysis results confirmed that the molecular multi-bilayer structure with $\beta$-sheet conformational chains successfully transformed back to an HCP structure with $8_{3}$ helical conformational chains by the retreatment with $\mathrm{CS}_{2}$ annealing (Figure 3).

The interesting chain conformations and morphological structures described above might result from selective and specific interactions of the PHIC polymer molecule with solvent molecules during the solvent-annealing process. We observed that $\mathrm{CHCl}_{3}$ is a good solvent for PHIC, where $\mathrm{CS}_{2}$ and toluene are relatively less good solvents. PHIC is composed of a relatively polar amide backbone and nonpolar $n$-hexyl side groups. $\mathrm{CHCl}_{3}$ is a polar solvent. Therefore, the polar $\mathrm{CHCl}_{3}$ molecule has a higher affinity for the amide units in the backbone than for the $n$-hexyl side groups. The favorable interaction of the solvent molecules with the amide backbone units can cause the rigid polymer backbones to mobilize, and then the mobilized backbones consequently cause their flexible $n$-hexyl side groups to mobilize. Owing to the good solvent characteristics and the polar nature, the $\mathrm{CHCl}_{3}$ molecule might interact more strongly with the amide backbone units, although the polymer backbone has considerable chain rigidity. As a result, the entire polymer chain is highly mobilized and consequently has less selectivity in its chain conformation and formation of its self-assembled structure, which leads to a mixture of two different conformations $\left(8_{3}\right.$ and $\left.2_{1}\right)$ and their own self-assembled structures in mixed phases (that is, a mixture of the hexagonal lattice structure with the $8_{3}$ chain conformation and the multi-bilayer structure with the $2_{1}$ chain conformation).

In contrast, toluene is a nonpolar solvent. Therefore, the nonpolar toluene has a higher affinity for the $n$-hexyl side groups than for the amide backbone. The favorable interaction of the solvent molecules and the $n$-hexyl side groups can cause the flexible alkyl side groups to mobilize. Then, the mobilized alkyl side groups subsequently try to direct the amide chain backbone to mobilize. However, this mobilization of the amide chain backbone might be limited to a certain degree because the polymer backbone has considerable chain rigidity and the toluene solvent has less good solvent characteristics and is nonpolar. Therefore, the limited mobilization of the polymer backbone may cause negative feedback to the mobility of the $n$-hexyl side groups. As a result, the overall mobility of the PHIC chain induced by toluene vapor is relatively lower. Specifically, the toluene-induced mobility of the polymer backbone is significantly less than that induced by $\mathrm{CHCl}_{3}$. Under this circumstance, the alkyl side groups appear to form a smectic A-like ordering rather than a well-defined lattice ordering or full disordering. This ordering subsequently induces the polar amide backbones that have a limited mobility to pack closely together via their electron donor and acceptor interactions. These interactions collectively lead the PHIC molecules to form a multi-bilayer structure with a $\beta$-sheet chain conformation.

$\mathrm{CS}_{2}$ is also a nonpolar solvent. In fact, in this molecule the sulfur atoms have relatively larger size (that is, volume), higher polarizability, higher electron affinity and higher electronegativity, compared those of the carbon atom. ${ }^{24}$ Furthermore, the sulfur atoms in the $\mathrm{CS}_{2}$ molecule are known to have weaker $\pi$-donor ability, which makes the carbon center atom more electrophilic. ${ }^{25,26}$ Therefore, each $\mathrm{C}=\mathrm{S}$ bond has a dipole moment, even though the magnitude of the dipole moment is low. These two $\mathrm{C}=\mathrm{S}$ bonds are linearly linked via the carbon center, and therefore, their molecule $\mathrm{CS}_{2}$ has no permanent dipole moment. Although $\mathrm{CS}_{2}$ is a nonpolar solvent, it presents locally weak polarity on each building element. Because of these characteristics, $\mathrm{CS}_{2}$ has a different chemical nature than toluene. Furthermore, these characteristics are known to make $\mathrm{CS}_{2}$ more reactive than carbon dioxide $\left(\mathrm{CO}_{2}\right){ }^{26-30}$ Owing to the overall nonpolar characteristic, the $\mathrm{CS}_{2}$ molecule might have a favorable level of affinity for the $n$-hexyl side groups. Because of the local polarities, the $\mathrm{CS}_{2}$ molecule might also have a favorable level of affinity for the amide backbone. The observed less good solvent characteristics suggest that the interactions of the alkyl side groups and the amide backbone with $\mathrm{CS}_{2}$ occur to a marginal degree. The interaction of the alkyl side groups with $\mathrm{CS}_{2}$ might be less than their interaction with toluene; however, the interaction of the amide backbone with $\mathrm{CS}_{2}$ might be greater than with toluene but considerably less than with $\mathrm{CHCl}_{3}$. Overall, the $\mathrm{CS}_{2}$ solvent molecule primarily induces both the rigid amide backbone and the $n$-hexyl side groups to mobilize in a proper level via favorable interactions in marginal levels; note that the mobility of the polymer backbone induced by $\mathrm{CS}_{2}$ appears to be greater than that induced in a secondary process by the nonpolar toluene but considerably less than that induced by the polar $\mathrm{CHCl}_{3}$. Such cooperative interactions collectively lead the PHIC molecules to have the $8_{3}$ helical conformations and form the HCP structure.

As discussed above, the chain conformation and formation of a self-assembled structure of the peptide-mimic rigid PHIC was well controlled by annealing under vapors of an appropriate solvent that 
can properly interact with the polymer chain. During the solventannealing process, it is very important to manage the specific interactions of a chosen solvent molecule with the polymer building components and their level in the appropriate way to provide the overall and local mobilities of the polymer chain required to form a certain chain conformation and its self-assembled structure.

\section{CONCLUSIONS}

For the first time, we succeeded in producing well-ordered PHIC structures with the aid of selective solvent-annealing, and we determined the details of their structures and conformations. PHIC formed a well-ordered HCP structure with $8_{3}$ helical conformational chains in the film that was annealed under vapors of $\mathrm{CS}_{2}$ (a less good solvent), which is a nonpolar solvent but has locally weak polarity on its building elements, and a well-ordered multi-bilayer structure with $2_{1}$ ( $\beta$-sheet) conformational chains in the film annealed under vapors of toluene, which is a nonpolar solvent and also another type of less good solvent. A fully reversible transformation between these two structures was observed by consecutive annealings with $\mathrm{CS}_{2}$ and toluene.

\section{CONFLICT OF INTEREST}

The authors declare no conflict of interest.

\section{ACKNOWLEDGEMENTS}

This study was supported by the National Research Foundation of Korea (Doyak project (2011-0028678) and the Center for Electro-Photo Behaviors in Advanced Molecular Systems (2010-0001784)), the Program for Integrated Molecular System of GIST and the Ministry of Education, Science and Technology (MEST) (BK21 Program and World Class University Program R31-2008-000-10059-0 and R31-20008-000-10026-0). The synchrotron X-ray scattering measurements at the Pohang Accelerator Laboratory were supported by MEST and POSCO Company, and POSTECH Foundation.

Author contributions: M.R. and J.-S.L. designed the research and initiated the study. J.M. and P.N.S. synthesized and characterized the material. Y.R., B.A., S.J., and K.K. conducted sample preparations and GIXS measurements. Y.R. and J.Y. did set up the theoretical GIXS framework and performed the data analyses. M.R., J.-S.L., Y.R., J.M., and J.Y. prepared the manuscript. All authors contributed to the discussion.

1 Bur, A. J. \& Fetters, L. J. The chain structure, polymerization, and conformation of polyisocyanates. Chem. Rev. 76, 727-746 (1976).

2 Vogl, O. \& Jaycox, G. D. Helical polymers. Polymer 28, 2179-2182 (1987).

3 Wulff, G. Main-chain chirality and optical activity in polymers consisting of C-C chains. Angew. Chem. 28, 21-37 (1989).

4 Okamoto, Y. \& Nakano, T. Asymmetric Polymerization. Chem. Rev. 94, 349-372 (1994).

5 Green, M. M., Peterson, N. C., Sato, T., Teramoto, A., Cook, R. \& Lifson, S. A helical poly mer with a cooperative response to chiral information. Science 268, 1860-1866 (1995).

6 Mayer, S. \& Zentel, R. Chiral polyisocyanates, a special class of helical polymers. Prog. Polym. Sci. 26, 1973-2013 (2001).

7 Fishwick, C. W. G., Beevers, A. J., Carrick, L. M., Whitehouse, C. D., Aggeli, A. \& Boden, N. Structures of helical $\beta$-tapes and twisted ribbons: the role of side-chain interactions on twist and bend behavior. Nano Lett. 3, 1475-1479 (2003).
8 Allenmark, S. Induced circular dichroism by chiral molecular interaction. Chirality 15, 409-422 (2003).

9 Tonelli, A. E. Conformational characteristics of the poly(n-alkyl isocyanates). Macromolecules 7, 628-631 (1974).

10 Han, C. C. -C. \& Yu, H. Chain configuration of poly(n-alkylisocyanate) with rotationa isomeric approximation. Polym. Prepr. 14, 121 (1973).

11 Troxell, T. C. \& Scheraga, H. A. Electric dichroism and polymer conformation. II. Theory of electric dichroism, and measurements on poly( $n$-butyl isocyanate). Macromolecules 4, 528-539 (1971).

12 Aharoni, S. M. Rigid backbone polymers. 2. Polyisocyanates and their liquid-crystal behavior. Macromolecules 12, 94-103 (1979).

13 Shmueli, U., Traub, W. \& Rosenheck, K. Structure of poly( $N$-butyl isocyanate). J. Polym. Sci. A-2 Polym. Phys. 7, 515-524 (1969).

14 Chen, J. T., Tomas, E. L., Ober, C. K. \& Hwang, S. S. Zigzag morphology of a poly(styrene- $b$-hexyl isocyanate) rod-coil block copolymer. Macromolecules $\mathbf{2 8}$, 1688-1697 (1995).

15 Burke, J. J. \& Weiss, V. Characterization of Materials in Research: Ceramics and Polymers 417-436 (Syracuse Univ., New York, USA, 1975).

16 Chen, J. T., Tomas, E. L., Ober, C. K. \& Mao, G -p. Self-assembled smectic phases in rod-coil block copolymers. Science 273, 343-346 (1996).

17 Shin, Y. -D., Kim, S. -Y., Ahn, J. -H. \& Lee, J. -S. Synthesis of poly(n-hexyl isocyanate) by controlled anionic polymerization in the presence of $\mathrm{NaBPh}_{4}$. Macromolecules 34, 2408-2410 (2001).

18 Ahn, J. -H., Shin, Y. -D. Nath, G. Y, Park, S. -Y, Rahman, M. S. Samal, S. \& Lee, J. -S. Unprecedented control over polymerization of $n$-hexyl isocyanate using an anionic initiator having synchronized function of chain-end protection. J. Am. Chem. Soc. 127 4132-4133 (2005).

19 Lee, B., Park, Y. -H., Hwang, Y. -T., Oh, W., Yoon, J. \& Ree, M. Ultralow-k nanoporous organosilicate dielectric films imprinted with dendritic spheres. Nat. Mater. 4, 147-150 (2005)

20 Yoon, J., Kim, K. -W., Kim, J., Heo, K., Jin, K. S., Jin, S., Shin, T. J., Lee, B., Rho, Y, Ahn, B. \& Ree, M. Small-angle X-ray scattering station 4C2 BL of Pohang accelerator laboratory for advance in Korean polymer science. Macromol. Res. 16, 575-585 (2008).

21 Lee, B., Park, I., Yoon, J., Park, S., Kim, J., Kim, K. -W., Chang, T. \& Ree, M. Structural analysis of block copolymer thin films with grazing incidence small angle $\mathrm{X}$-ray scattering. Macromolecules 38, 4311-4323 (2005).

22 Ahn, B., Hirai, T., Jin, S., Rho, Y., Kim, K. -W., Kakimoto, M. -a., Gopalan, P., Hayakawa, T. \& Ree, M. Hierarchical structure in nanoscale thin films of a poly(styreneb-methacrylate grafted with POSS) (PS214-b-PMAPOSS27). Macromolecules 43 10568-10581 (2010).

$23 \mathrm{Kim}$, G., Yoon, J., Kim, J. -S., Kim, H. \& Ree, M. Molecular fibers based on the honeycomb-like self-assembly of an alpha-helical polypeptide. J. Phys. Chem. B 112 8868-8870 (2008)

24 Oxtoby, D. W., Gillis, H. P. \& Campion, A. Principles of Modern Chemistry. 7th Edn Chapter 3 (Brooks/Cole, Pacific Grove, California, USA, 2012).

25 Iglesias-Sigüenza, F. J. Carbon disulfide (CS $)$. Synlett 157-158 (2009).

26 Rudorf, W. -D. Reactions of carbon disulfide with N-nucleophiles. J. Sulfur Chem. 28 295-339 (2007)

27 Lee, B., Jung, J. H. \& Ree, M. Synthesis and characterization of poly(propylene sulfite) from sulfur dioxide and propylene oxide using organometallic catalysts. Macromol. Chem. Phys. 201, 831-839 (2000).

28 Ree, M., Bae, J. Y., Jung, J. H. \& Shin, T. J. A new copolymerization process leading to poly(propylene carbonate) with a highly enhanced yield from carbon dioxide and propylene oxide. J. Polym. Sci. A Polym. Chem. 37, 1863-1876 (1999).

29 Hwang, Y., Jung, J., Ree, M. \& Kim, H. Terpolymerization of $\mathrm{CO}_{2}$ with propylene oxide and caprolactone using zinc glutarate catalyst. Macromolecules 36, 8210-8212 (2003).

30 Ree, M., Bae, J. Y., Jung, J. H. \& Shin, T. J. Copolymerization of carbon dioxide and propylene oxide using various zinc glutarate derivatives as catalysts. Polym. Eng. Sci. 40, 1542-1552 (2000)

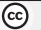

This work is licensed under the Creative Commons Attribution-NonCommercial-No Derivative Works 3.0 Unported License. To view a copy of this license, visit http:// creativecommons.org/licenses/by-nc-nd/3.0/

Supplementary Information accompanies the paper on the NPG Asia Materials website (http://www.nature.com/am) 